\section{LA IMPORTANCIA DEL AGUA EN LA INDUSTRIA DE ALIMENTOS VEGETALES}

\author{
Francisco López-Gálvez \\ Centro de Edafología y Biología Aplicada del Segura (CEBAS) \\ Consejo Superior de Investigaciones Científicas \\ ORCID iD: https://orcid.org/0000-0002-4861-5878 \\ flopez@cebas.csic.es \\ Maria Isabel Gil \\ Centro de Edafología y Biología Aplicada del Segura (CEBAS) \\ Consejo Superior de Investigaciones Científicas \\ ORCID iD: https://orcid.org/0000-0003-4340-7727 \\ migil@cebas.csic.es
}

Cómo citar este artículo/Citation: López-Gálvez, F. y Gil, M. I. (2020). La importancia del agua en la industria de alimentos vegetales. Arbor, 196 (795): a547. https://doi.org/10.3989/ arbor.2020.795n1011

Recibido: 26 febrero 2019. Aceptado: 29 octubre 2019.

RESUMEN: La industria de alimentos vegetales consume grandes volúmenes de agua de buena calidad y genera grandes cantidades de agua residual. Uno de los sistemas que se pueden aplicar para reducir el consumo y el vertido de agua es la reutilización del agua de lavado. Para llevar a cabo la reutilización del agua sin comprometer la seguridad microbiológica y química de los alimentos es necesario optimizar el uso de agentes antimicrobianos. El cloro ha sido tradicionalmente el tratamiento de desinfección usado por la industria hortofrutícola para el agua de lavado. Sin embargo, su uso tiene la desventaja de la acumulación de subproductos de desinfección, lo que ha impulsado el estudio y el uso de desinfectantes alternativos como el ácido peroxiacético. En cualquier caso, el mantenimiento de la seguridad microbiológica y química de los alimentos vegetales frescos, en lo que respecta a la etapa de lavado, pasa por la selección de los límites operacionales que deben mantenerse en cuanto a la concentración de desinfectante, así como la monitorización y control de los parámetros críticos relacionados con la desinfección, como es el contenido en materia orgánica. La optimización de la desinfección tiene que tener en cuenta las peculiaridades de cada caso, incluyendo el tipo de producto lavado, el desinfectante usado, y el diseño y manejo de la línea de lavado.

PALABRAS CLAVE: vegetales frescos; agua de lavado; seguridad; calidad; contaminación cruzada.

\section{THE IMPORTANCE OF WATER IN THE FRESH PRODUCE INDUSTRY}

Copyright: (C) 2020 CSIC. Este es un artículo de acceso abierto distribuido bajo los términos de la licencia de uso y distribución Creative Commons Reconocimiento 4.0 Internacional (CC BY 4.0).

ABSTRACT: The fresh produce industry consumes large volumes of good quality water and generates large amounts of wastewater. One of the systems that can be applied to reduce water consumption and water discharge is the reuse of washing water. To carry out the reuse of water without compromising the microbiological and chemical safety of the food, it is necessary to optimize the use of antimicrobial agents. Chlorine has traditionally been the disinfection treatment used by the horticultural industry for washing water. However, its use has the disadvantage of accumulating disinfection byproducts, which has led to the study and use of alternative disinfectants such as peroxyacetic acid. In any case, maintaining the microbiological and chemical safety of fresh produce in the washing stage involves the selection of operational limits that must be maintained (e.g., the concentration of disinfectant), as well as the monitoring and control of critical parameters related to disinfection such as the content of organic matter. The optimization of disinfection must take into account the peculiarities of each case, including the type of product washed, the disinfectant used and the design and management of the washing line.

KEYWORDS: fruits and vegetables; wash water; safety; quality; cross-contamination. 


\section{EL AGUA EN LA INDUSTRIA DE ALIMENTOS VEGETA- LES (EL AGUA DE LAVADO Y SU REUTILIZACIÓN)}

El agua tiene diferentes usos en las plantas de acondicionamiento y procesado mínimo de la industria de alimentos vegetales frescos, ya que se utiliza para transportar el producto entre zonas en la planta, para enfriar el producto a través del contacto directo con agua fría, y para eliminar la suciedad de campo en la superficie del mismo (véase figura 1) (véase Whitaker, Key Learnings from CPS Wash Water Symposium). Esta industria tiene una alta demanda de agua y genera grandes cantidades de aguas residuales o de vertido (Manzocco et al., 2015, p. 286; Ölmez y Kretzschmar, 2009, p. 686). Para poder reducir esta gran demanda de agua, se necesitan estrategias que, al mantener su calidad química y microbiológica, permitan su reutilización (Casani, Rouhany y Knøchel, 2005, p. 1134; Gil, Selma, López-Gálvez y Allende, 2009, p. 37). La información existente sobre el papel del agua de lavado con respecto a las enfermedades transmitidas por los alimentos es escasa, y el impacto del agua como ingrediente en el producto alimenticio fabricado ha sido ignorado (Mena, 2006, p. 95). Recientemente la calidad del agua se está reconociendo como un aspecto

Figura 1. Línea de lavado industrial de pimiento entero

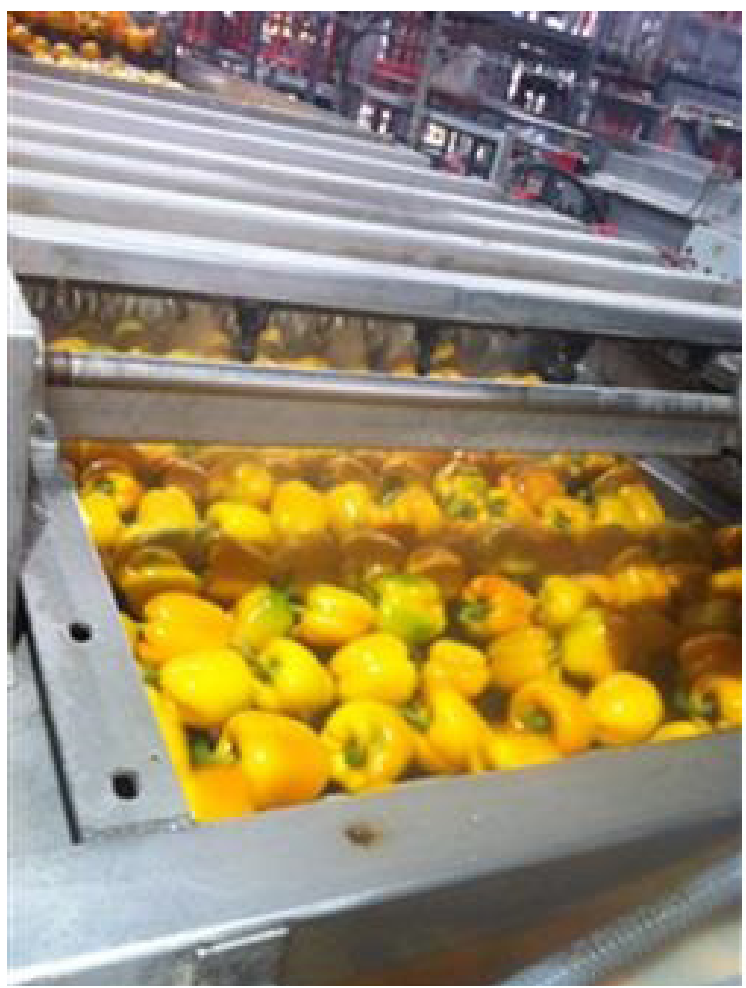

Fuente: elaboración propia. importante que afecta directamente a la inocuidad de los alimentos durante la producción y el procesado, particularmente para productos vegetales que se consumen sin tratamiento térmico previo (Gil et al., 2009, p. 37; Gombas et al., 2017, p. 312). La calidad del agua es aún más crítica en la industria de IV Gama, en la que todos los productos se lavan después del corte para eliminar los exudados del producto cortado (USFDA, 2001). El proceso de corte expone tejido que normalmente está protegido por barreras de defensa naturales como la cutícula y la epidermis, por ejemplo, y el agua que entra en contacto directo con el tejido interno de los alimentos vegetales.

En cuanto al marco legislativo, en la Unión Europea debe usarse agua potable para el lavado final de los alimentos vegetales frescos listos para el consumo, mientras que puede usarse agua limpia para las etapas iniciales de lavado (véase Reglamento (CE) $n^{\circ}$ 852/2004 del Parlamento Europeo y del Consejo, de 29 de abril de 2004, relativo a la higiene de los productos alimenticios, p. 1; Nota de la Comisión sobre la Guía para combatir los riesgos microbiológicos en frutas y hortalizas frescas en la producción primaria mediante una buena higiene, p. 1). El agua potable sería

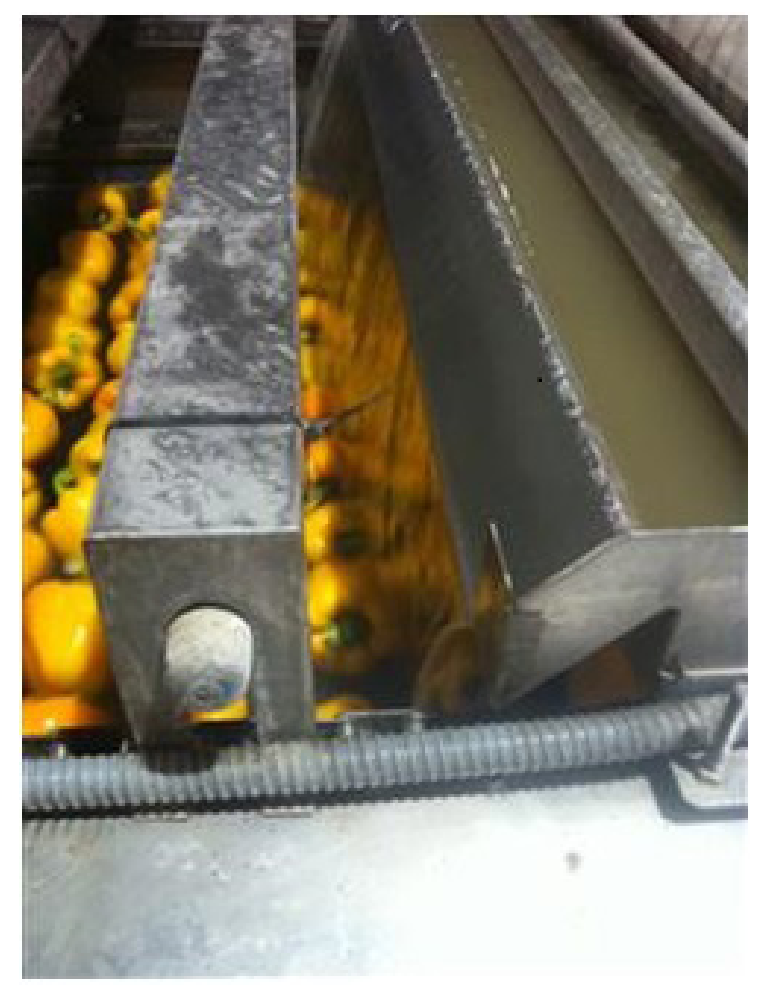


el agua que cumple los requisitos mínimos establecidos en la Directiva 98/83/CE del Consejo de 3 de noviembre de 1998 relativa a la calidad de las aguas destinadas al consumo humano (véase Directiva 98/83/ CE del Consejo de 3 de noviembre de 1998 relativa a la calidad de las aguas destinadas al consumo humano, p. 32). Por otro lado, el agua limpia sería agua natural, artificial o purificada que no contenga microorganismos ni sustancias nocivas en cantidades que puedan afectar directa o indirectamente a la calidad sanitaria de los productos alimenticios (véase Reglamento (CE) $\mathrm{n}^{\circ}$ 852/2004 del Parlamento Europeo y del Consejo, de 29 de abril de 2004, relativo a la higiene de los productos alimenticios, p. 1). Con el fin de reducir la demanda de agua, en Bélgica la Agencia Federal para la Seguridad de la Cadena Alimentaria permite el uso de agua limpia o reciclada durante las etapas iniciales de lavado de frutas y hortalizas (Holvoet, Jacxsens, Sampers y Uyttendaele, 2012, p. 671).

Existen numerosos estudios que han puesto de manifiesto la importancia del uso de agentes antimicrobianos en el agua de lavado para prevenir la contaminación cruzada (Gombas et al., 2017, p. 312). La Comisión Europea contempla la utilización de agentes antimicrobianos en el agua de proceso como una de las estrategias que se pueden usar para mantener la calidad del agua y evitar la contaminación cruzada (véase Nota de la Comisión sobre la Guía para combatir los riesgos microbiológicos en frutas y hortalizas frescas en la producción primaria mediante una buena higiene, p. 1). Sin embargo, la eficacia de estos tratamientos antimicrobianos puede verse afectada por varios factores, principalmente la suciedad proveniente del campo de cultivo (polvo, tierra, etc.) y el contenido en materia orgánica (restos vegetales y exudados del corte o de zonas dañadas del producto) (LópezGálvez et al., 2009, p. 167). Hasta ahora, el cloro ha sido el desinfectante de elección en la industria hortofrutícola. Sin embargo, su asociación a subproductos de desinfección tales como trihalometanos y clorato han impulsado la búsqueda de alternativas a su uso.

\section{EL AGUA EN LA INDUSTRIA DE ALIMENTOS VEGETALES Y SUS IMPLICACIONES EN LA SEGURIDAD ALIMENTARIA}

Las investigaciones llevadas a cabo en productos vegetales frescos han demostrado que el uso de agua de lavado contaminada con microorganismos patógenos ha contribuido a la aparición de brotes de toxiinfecciones alimentarias (Gombas et al., 2017, p. 312). Por lo tanto, un agua de lavado de mala calidad es uno de los factores de riesgo que afecta directamente a la se- guridad de las frutas y hortalizas, incluyendo los productos de IV Gama. La principal función de los agentes antimicrobianos usados durante el lavado es evitar la transferencia de microorganismos patógenos entre producto contaminado y producto no contaminado a través del agua de lavado (Gil et al., 2009, p. 37).

El agua de lavado de frutas y hortalizas presenta altas concentraciones de materia orgánica, incluidos exudados del producto vegetal y microorganismos. Las características del agua cambian constantemente durante el lavado de frutas y hortalizas (López-Gálvez, Tudela, Allende y Gil, 2019, p. 211; Selma et al., 2008a, p. 286) debido a una serie de causas entre las que se incluyen:

- Cambios en el tipo de producto lavado o de las características del mismo producto entre lotes.

- Cambios en la velocidad del flujo de producto en su paso por la etapa de lavado.

- Cambios en los caudales de entrada y salida de agua de los tanques de lavado.

- Aplicación puntual de productos químicos para desinfección y para regulación del $\mathrm{pH}$.

Durante el lavado el tanque se rellena constantemente con agua para incorporar la que se pierde en la superficie del producto. Sin embargo, esta renovación parcial del agua de lavado no es suficiente para evitar la acumulación de materia orgánica, incluyendo la flora microbiana de la superficie del vegetal. Las características físico-químicas del agua de lavado juegan un papel muy importante en la eficacia del tratamiento antimicrobiano aplicado. Diferentes estudios han demostrado que la presencia de una elevada concentración de materia orgánica en el agua de lavado dificulta la inactivación de los microorganismos (véase figura 2) (López-Gálvez et al., 2012, p. 146; Van Haute et al., 2015, p. 102).

Asociada al uso de agentes antimicrobianos se encuentra la acumulación de subproductos de desinfección en el agua de lavado y en el tejido vegetal, que constituyen un riesgo para la salud del consumidor (Gil, Marín, Andujar y Allende, 2016, p. 416). Los subproductos de desinfección mejor conocidos son aquellos derivados del uso de agentes antimicrobianos basados en el cloro, como los trihalometanos (THMs) y los ácidos haloacéticos (HAAs), como compuestos orgánicos, y el clorato como subproducto inorgánico. En concreto, la presencia de altas concentraciones de clorato en los alimentos vegetales está causando grandes pérdidas a las empresas productoras, ya que existen lí- 
Figura 2. Inactivación de Escherichia coli 0157:H7 mediante la desinfección electroquímica de agua de proceso con diferentes niveles de demanda química de oxígeno (DQO) de lechuga IV Gama

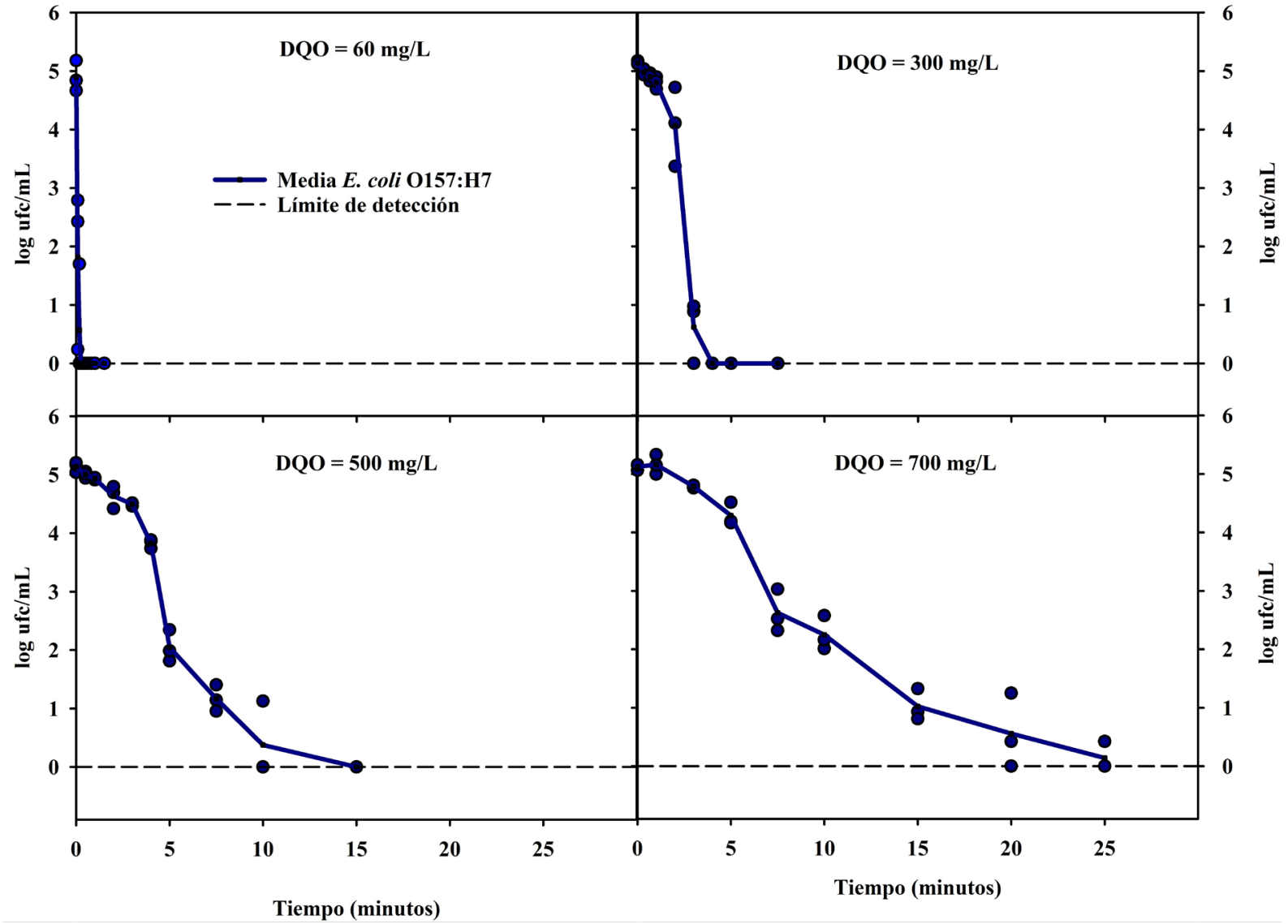

Fuente: López-Gálvez et al., 2012, p. 146.

mites máximos autorizados por la Unión Europea, por encima de los cuales estos productos son rechazados (Gil et al., 2016, p. 416). En un estudio llevado a cabo en Alemania, se detectó clorato en diferentes tipos de frutas y hortalizas en concentraciones muy superiores al límite máximo residual (LMR) establecido por la Legislación Europea en 0,01 mg/kg (Kaufmann-Horlacher, Scherbaum, Stroher-Kolberg y Wildgrube, 2014). De las 1.087 muestras de alimentos vegetales frescos analizadas, un $24,5 \%$ (266) presentaban residuos de clorato en una concentración superior a la permitida. En dicho estudio, el uso de agentes antimicrobianos basados en el cloro en el lavado de frutas y hortalizas, fue señalado como una de las posibles causas de los niveles de clorato detectados. El panel de expertos en contaminantes de la European Food Safety Authority (EFSA) analizó los resultados de 6.359 análisis de clorato en muestras de frutas y hortalizas realizados en toda la Unión Europea (EFSA, 2015, p. 4135). En un $22 \%$ de dichas muestras se detectaron niveles de cloratos por encima del límite de detección. En base a dichos resultados se pudo concluir que utilizando las prácticas agronómicas y de procesado habituales, se alcanzan concentraciones de clorato por encima de los niveles autorizados en muchos productos vegetales. Por ello, la EFSA a través de cada estado miembro está recopilando datos sobre los niveles encontrados antes de regular el LMR especifico de cada producto.

Para reducir el riesgo de contaminación cruzada por microorganismos patógenos y la presencia de subproductos de desinfección se deben fijar los límites operacionales respecto a la concentración de desinfectante y seleccionar aquellos parámetros críticos de la etapa de lavado que deben medirse para llevar a cabo una monitorización y control adecuada de la desinfección. Diversos estudios han evaluado diferentes tecnologías alternativas al uso de agentes antimicrobianos químicos para el tratamiento del agua de lavado de frutas y hortalizas, como por ejemplo el 
uso combinado de ultrasonidos y luz ultravioleta. Sin embargo, el tratamiento con agentes químicos sigue siendo el tipo de tratamiento más utilizado. El sistema de control debe permitir mantener una concentración de agente antimicrobiano lo suficientemente baja para evitar la contaminación cruzada, y lo suficientemente alta para evitar la acumulación de subproductos de desinfección.

\section{EL AGUA EN LA INDUSTRIA DE ALIMENTOS VEGETALES FRESCOS Y SUS IMPLICACIONES MEDIOAMBIENTALES}

Las plantas en las que se realiza el lavado de frutas y hortalizas generan grandes volúmenes de aguas residuales que contienen elevados niveles de materia orgánica, así como microorganismos y contaminantes químicos (Manzocco et al., 2015, p. 286; Ölmez y Kretzschmar, 2009, p. 686). Se han realizado grandes esfuerzos en el desarrollo de estrategias para reducir el volumen de agua residual con la recuperación de gran parte de su volumen para volver a utilizarlo en el proceso de lavado.

Las tecnologías para el tratamiento del agua que se pueden aplicar directamente en el tanque de lavado y las que se pueden aplicar fuera de la línea son diferentes. Esto se debe a que en el tanque de lavado existe un contacto directo con el producto. Si se lleva a cabo un tratamiento adecuado fuera de la línea, el agua se puede introducir de nuevo en el sistema para ser reutilizada otra vez en el lavado de producto (Gómez-López, Gil, Allende, Vanhee y Selma, 2015, p. 29; Van Haute et al., 2015, p. 102). Sin embargo, el tratamiento del agua de proceso fuera de la línea para su reutilización no es frecuente, ya que estas tecnologías son muy costosas y de difícil mantenimiento, por lo que se debe justificar su empleo para poder implementarlas. Entre las razones que justificarían su uso estarían, por ejemplo, un alto coste de los vertidos, una escasez de agua, o el que los vertidos sean muy contaminantes como por ejemplo aquellos con un gran contenido de materia orgánica que deben ser tratados antes de ser vertidos. En el caso del tratamiento de agua fuera de la línea para poder ser reutilizada, la velocidad de inactivación de los microorganismos no es un parámetro tan crucial, ya que los tiempos de contacto pueden ser más largos. En este caso, a la hora de calcular la dosis de los desinfectantes químicos, no hay que tener en cuenta el daño en el producto, pero se deben usar concentraciones mínimas efectivas para evitar la generación excesiva de subproductos de desinfección. Las tecnologías de filtrado que permiten reducir la materia orgánica y los restos vegetales pueden ayudar a que los desinfectantes sean más eficaces. Asimismo, mediante floculación, coagulación y eliminación por centrifugación para posteriormente pasar a través de sistemas de filtración por ósmosis inversa y tratamiento UV, se puede obtener un agua con una calidad microbiológica de agua limpia para poder ser de nuevo empleada en la planta de procesado. La empresa Simply Clean Technologies. (https://simplycleantechnologies.com/technology/) ha creado soluciones de este tipo para las aguas de proceso de vegetales frescos. La tecnología de Simply Clean Technologies permite el lavado de los productos vegetales usando duchas con agua tratada recirculada (Bornhorst et al., 2018, p. 124). Este sistema tiene una serie de ventajas sobre el sistema más común de inmersión en tanques de lavado. Al no haber inmersión de producto en la misma agua, es difícil la contaminación cruzada a través del agua. Además, el agua usada para rociar el producto se recoge y se trata fuera de la línea antes de volver a utilizarse con el fin de evitar riesgos microbiológicos y químicos (véase figura 3). Con ello, a diferencia de lo que ocurre en la reutilización directa en el tanque de lavado, se evita el deterioro de la calidad del agua de lavado que entra en contacto con el producto (p. ej., la cantidad de materia orgánica en el agua de lavado se mantiene estable).

Figura 3. Agua de lavado de vegetales antes del tratamiento (izquierda), y agua de lavado de vegetales tratada con el sistema de Simply Clean Technologies (derecha)

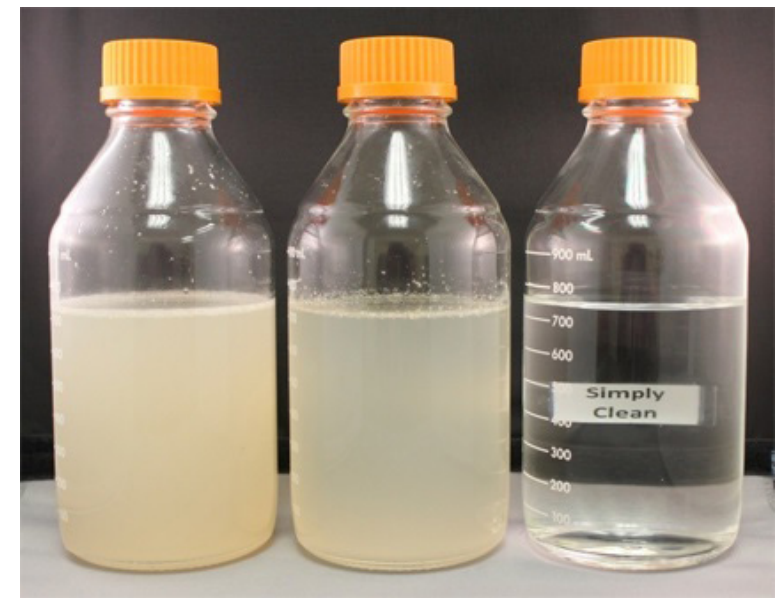

Fuente: https://simplycleantechnologies.com/technology/

\section{VISIÓN GENERAL DE LA EVOLUCIÓN DE LOS TRATA- MIENTOS DE DESINFECCIÓN A LO LARGO DEL TIEM- PO Y PERSPECTIVAS DE FUTURO}

La desinfección es la eliminación o inactivación de microorganismos patógenos mediante métodos físicos (por ejemplo, luz UV, ultrasonidos, filtración) o químicos (por ejemplo, cloro, ozono). Existe informa- 
ción sobre multitud de tecnologías de desinfección aplicadas en vegetales frescos a escala de laboratorio. Sin embargo, en muchos casos, la eficacia durante el lavado industrial de vegetales no ha sido verificada. Para evitar la contaminación cruzada, la velocidad de inactivación de los microorganismos (por ejemplo, ufc/mL-min) por parte del sistema de desinfección usado es el parámetro que hay que tener en cuenta. Cuanto mayor sea esta velocidad, más seguro es que se inactivarán los microorganismos patógenos antes de poder transferirse al producto no contaminado. Para un desinfectante químico, habría que calcular la concentración necesaria que debe mantenerse en el agua para que la inactivación se produzca de forma casi instantánea. La distribución homogénea del desinfectante en el tanque de lavado también es importante, para evitar que haya zonas donde la concentración del desinfectante sea inferior a la necesaria. Además, el desinfectante no debe causar deterioro de la calidad del producto.

Los desinfectantes con potencial aplicación para el agua de lavado de frutas y hortalizas a escala industrial han ido poco a poco evolucionando fundamentalmente en lo que respecta a las concentraciones efectivas y a los parámetros de medida y control. El cloro, principalmente en forma de hipoclorito de sodio ( $\mathrm{NaClO}$ ), es el desinfectante más común en el lavado de frutas y hortalizas (Fu, Li, Awad, Zhou y Liu, 2018, p. 2012). Este agente es utilizado en el agua de los tanques de lavado, en los canales de descarga y transporte del producto y en las duchas en las plantas hortofrutícolas. Tanto si se utiliza el hipoclorito de sodio como si se usa otra fuente de cloro (por ejemplo, hipoclorito de calcio o cloro gas), al añadirlo al agua se forma ácido hipocloroso $(\mathrm{HClO})$ más el ion hipoclorito $\left(\mathrm{ClO}^{-}\right)$ y cloro molecular $\left(\mathrm{Cl}_{2}\right)$, la suma de todos ellos es el cloro libre. Independientemente de la concentración de cloro inicial y de la carga orgánica presentes en el agua de lavado, el parámetro clave que determina la capacidad de inactivación de microorganismos es la concentración de cloro libre residual. El agente más activo desde el punto de vista antimicrobiano es el ácido hipocloroso. La relación entre la concentración de $\mathrm{HClO}_{\text {y de }} \mathrm{ClO}^{-}$va a depender en gran parte del $\mathrm{pH}$, alcanzándose la máxima concentración de ácido hipocloroso en el intervalo de pH entre 5,0 y 6,5. El cloro reacciona con compuestos nitrogenados orgánicos presentes en las aguas de lavado (por ejemplo, aminoácidos y proteínas). Estos compuestos reducen la cantidad de cloro libre y forman cloraminas orgánicas, que tienen poco o ningún efecto antimicrobiano. En muchas ocasiones, las empresas usan dosis de cloro innecesariamente altas (hipercloración), manteniendo concentraciones residuales de cloro libre superiores a $100 \mathrm{mg} / \mathrm{L}$. Para evitar la hipercloración, sería necesario que, para cada aplicación (definida por factores como el tipo de producto, el flujo de producto o el diseño de la etapa de lavado), se llevara a cabo la optimización de forma que se empleen concentraciones mínimas. El principal factor que puede hacer bajar la concentración de cloro libre por debajo de los niveles deseados es la acumulación de materia orgánica disuelta en el agua de lavado. En el caso del lavado de hortalizas de hoja se ha propuesto una concentración residual de cloro libre de $2-10 \mathrm{mg} / \mathrm{L}$ a un $\mathrm{pH}$ adecuado $(5,0-6,5)$ como la mínima necesaria para mantener bajos niveles de microorganismos en el agua de lavado (Gómez-López, Lannoo, Gil y Allende, 2014, p. 132; Luo et al., 2011, p. 352; Munther y Wu, 2013, p. 28). Para evitar que debido a picos en la demanda de cloro haya momentos en que no quede suficiente cloro libre en el agua, se deberían utilizar concentraciones en la zona alta de dicho intervalo $(10 \mathrm{mg} / \mathrm{L})$. Se debe medir el nivel de desinfectante de forma rigurosa y con regularidad. En la actualidad, la industria hortofrutícola todavía tiene un amplio margen de mejora en cuanto a la monitorización de la concentración de desinfectantes para asegurar una acción antimicrobiana eficaz y minimizar la presencia de subproductos de desinfección (López-Gálvez et al., 2019, p. 211). Empresas punteras como Smartwash Solutions ${ }^{\mathrm{TM}}$ han desarrollado un sistema que permite controlar de manera precisa la concentración de cloro libre residual y el $\mathrm{pH}$ del agua de lavado, lo que permite ajustar la concentración de cloro al mínimo necesario para evitar la contaminación cruzada. Otras empresas como Palintest comercializan los equipos para la monitorización de la concentración de cloro libre y de cloro total por medio de sensores electroquímicos (véase figura 4), lo que ayuda a calibrar y confirmar el correcto funcionamiento de los sensores on line para asegurar la calidad del agua y evitar la contaminación cruzada.

El agua electrolizada es una tecnología de desinfección basada en el cloro con una serie de ventajas sobre otros derivados clorados. Para su producción solo se necesita $\mathrm{NaCl}$ y agua por lo que, tras la inversión inicial, el gasto que conlleva es en electricidad (Hricova, Stephan y Zweifel, 2008, p. 1934). Su eficacia es mayor que la del hipoclorito de sodio a igual concentración de cloro libre (Abadias, Usall, Oliveira, Alegre, Viñas, 2008, p. 151). Además, al producirse in situ, la solución desinfectante no se almacena por largos periodos de tiempo, lo que reduce la formación de clorato. Por desgracia, los resultados que respalden su 
Figura 4. Equipo Chlorosense HR de Palintest para la medida de cloro libre (hasta $25 \mathrm{mg} / \mathrm{L}$ ) y cloro total (hasta $500 \mathrm{mg} / \mathrm{L}$ ).

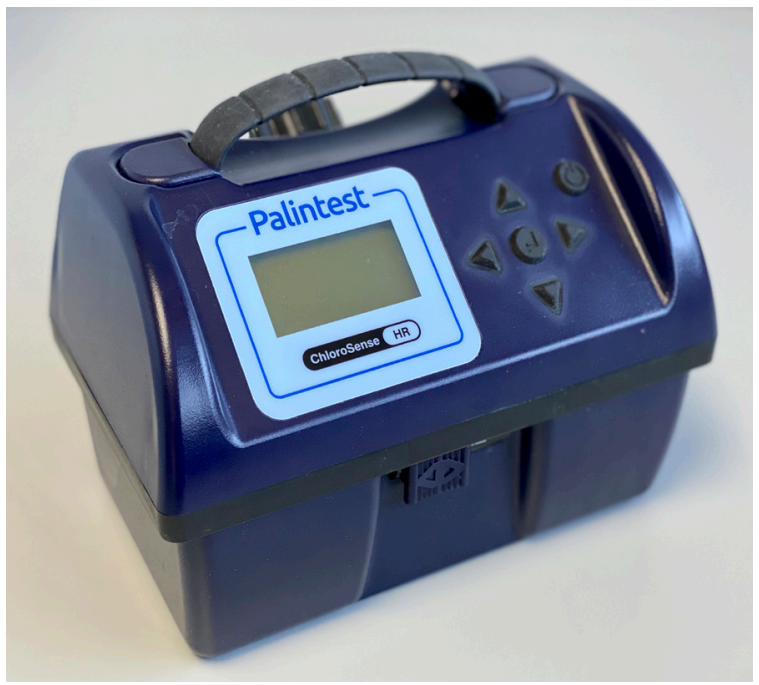

Fuente: elaboración propia.

eficacia para el agua de lavado durante el procesado a escala industrial de vegetales son escasos. Hay que tener en cuenta que, al ser una tecnología de desinfección basada en el cloro, la presencia de materia orgánica afecta a la eficacia de la desinfección, y durante su uso también se forman y acumulan subproductos de desinfección clorados.

El dióxido de cloro $\left(\mathrm{ClO}_{2}\right)$ es otro desinfectante con alta capacidad de inactivación de microorganismos, aunque los mecanismos de desinfección y de formación de subproductos son distintos a los del ácido hipocloroso. Entre sus ventajas está el hecho de que no da lugar a la formación de trihalometanos en sus reacciones con la materia orgánica (López-Gálvez et al., 2010, p. 53). Otra ventaja es su efectividad en un amplio rango de $\mathrm{pH}$ (entre 5 y 9 ). Sin embargo, el uso del dióxido de cloro como desinfectante da lugar a la acumulación de subproductos de desinfección inorgánicos como clorito, clorato, y perclorato (véase Chlorine and its oxides: Chlorate and perchlorate review).

El ácido peroxiacético (PAA) también es un desinfectante efectivo para el tratamiento del agua de lavado. Entre sus ventajas respecto al cloro están el menor impacto que tiene la presencia de materia orgánica en su eficacia. Además, la mayoría de los subproductos derivados de su uso son inofensivos ya que deja como principales residuos ácido acético, agua y oxígeno (Kitis, 2004, p. 47). Sin embargo, su velocidad de inactivación de microorganismos es inferior a la del cloro (Van Haute et al., 2015, p. 102). El PAA comercial es una mezcla de PAA, ácido acético y peróxido de hidrógeno, aunque la actividad antimicrobiana se debe principalmente al PAA. El control del pH no es tan crucial como en el caso del cloro, aunque su eficacia aumenta a pH más ácidos (Van Haute et al., 2015, p. 102). Entre sus desventajas estaría su elevado coste en comparación con otros desinfectantes como los hipocloritos de sodio y de calcio.

La radiación ultravioleta (UV) también se puede usar para el tratamiento del agua de lavado de alimentos vegetales (Selma, Allende, López-Gálvez, Conesa y Gil, 2008b, p. 809). Sin embargo, su acción antimicrobiana se ve afectada por la turbidez del agua, el color y la presencia de partículas. Además, su acción no tiene efecto residual en el agua. Para asegurar su eficacia, son importantes las pautas para la instalación y el uso de los equipos de radiación UV. Su uso combinado con sistemas de filtración puede evitar el efecto de la turbidez y mejorar el proceso de desinfección del agua de proceso.

\section{CONCLUSIONES}

El lavado de las frutas y hortalizas frescas y mínimamente procesadas que realiza la industria hortofruticola genera un gran volumen de aguas de proceso las cuales presentan un alto contenido en materia orgánica, y en las que hay una acumulación de microorganismos y de subproductos de desinfección. Con el objetivo de sostenibilidad para reducir la demanda de agua y la generación de un gran volumen de agua residual, una de las estrategias que se pueden aplicar es la reutilización del agua de lavado. Sin embargo, cuando se lleva a cabo la reutilización del agua, la entrada de producto contaminado con microorganismos patógenos en el agua de lavado supone un riesgo de amplificación de la contaminación por transferencia de microorganismos a otros lotes. Si no se lleva a cabo un tratamiento antimicrobiano eficaz, se puede producir contaminación cruzada entre lotes de producto contaminados y lotes no contaminados. Por otro lado, si se utilizan los desinfectantes en dosis excesivas, se puede generar un riesgo químico por la formación y acumulación de subproductos. Uno de los parámetros que más afectan a la calidad del agua de lavado de alimentos vegetales es la concentración de materia orgánica. Este parámetro afecta a la eficacia de los desinfectantes y a la formación y acumulación de subproductos de desinfección. Se deben establecer los límites operaciones entre los cuales debe regularse la concentración del desinfectante, así como seleccionar 
aquellos parámetros críticos que permitan el seguimiento y control del proceso de desinfección del agua de lavado de vegetales frescos. Se ha de realizar una monitorización y un control del proceso integral para que la concentración del desinfectante y las características fisicoquímicas del agua se encuentren siempre dentro de los límites operacionales que permitan evi- tar los riesgos microbiológicos y químicos asociados a la calidad del agua de lavado de vegetales frescos.

\section{AGRADECIMIENTOS}

Los autores agradecen la financiación del Center for Produce Safety (Proyecto 2017-01) y del MINECO (Proyecto AGL2016-75878-R).

\section{BIBLIOGRAFÍA}

Abadias, M., Usall, J., Oliveira, M., Alegre, I., Viñas, I. (2008). Efficacy of neutral electrolyzed water (NEW) for reducing microbial contamination on minimallyprocessed vegetables. International Journal of Food Microbiology, 123, pp. 151-158. https://doi.org/10.1016/j. ijfoodmicro.2007.12.008

Bornhorst, E. R., Luo, Y., Park, E., Vinyard, B. T., Nou, X., Zhou, B., Turner, E. y Millner, P. D. (2018). Immersion-free, singlepass, commercial fresh-cut produce washing system: An alternative to flume processing. Postharvest Biology and Technology, 146, 124-133. https://doi. org/10.1016/j.postharvbio.2018.08.008

Casani, S., Rouhany, M. y Knøchel, S. (2005). A discussion paper on challenges and limitations to water reuse and hygiene in the food industry. Water Research, 39, pp. 1134-1146. https://doi. org/10.1016/j.watres.2004.12.015

European Food Safety Authority (EFSA) (2015). Risks for public health related to the presence of chlorate in food. EFSA Panel on Contaminants in the Food Chain (CONTAM). EFSA Journal, 13 (6), 4135. https://doi.org/10.2903/j. efsa.2015.4135

Fu, T.-J., Li, Y., Awad, D., Zhou, T.-Y. y Liu, L. (2018). Factors affecting the performance and monitoring of a chlorine wash in preventing Escherichia coli 0157:H7 cross-contamination during postharvest washing of cut lettuce. Food Control, 94, pp. 212-221. https://doi.org/10.1016/j. foodcont.2018.06.035

Gil, M. I., Selma, M. V., López-Gálvez, F. y Allende, A. (2009). Fresh-cut product sanitation and wash water disinfection: Problems and solutions. International Journal of Food Microbiology, 134 (1-2), pp. 37-45. https://doi.org/10.1016/j. ijfoodmicro.2009.05.021

Gil, M. I., Marín, A., Andujar, S. y Allende, A. (2016). Should chlorate residues be of concern in fresh-cut salads? Food
Control, 60, pp. 416-421. https://doi. org/10.1016/j.foodcont.2015.08.023

Gombas, D., Luo, Y., Brennan, J., Shergill, G., Petran, R., Walsh, R., Hau, H., Khurana, K., Zomorodi, B., Rosen, J., Varley, R. y Deng, K. (2017). Guidelines to validate control of cross-contamination during washing of fresh-cut leafy vegetables. Journal of Food Protection, 80, pp. 312-330. https://doi. org/10.4315/0362-028X.JFP-16-258

Gómez-López, V. M., Lannoo, A.-S., Gil, M. I. y Allende, A. (2014). Minimum free chlorine residual level required for the inactivation of Escherichia coli O157:H7 and trihalomethane generation during dynamic washing of freshcut spinach. Food Control, 42, pp. 132-138. https://doi.org/10.1016/j.foodcont.2014.01.034

Gómez-López, V. M., Gil, M. I., Allende, A., Vanhee, B. y Selma, M. V. (2015). Water reconditioning by high power ultrasound combined with residual chemical sanitizers to inactivate foodborne pathogens associated with fresh-cut products. Food Control, 53, 29-34. https://doi. org/10.1016/j.foodcont.2014.12.032

Holvoet, K., Jacxsens, L., Sampers, I. y Uyttendaele, M. (2012). Insight into the prevalence and distribution of microbial contamination to evaluate water management in the fresh produce processing industry. Journal of Food Protection, 75, 671-681. https://doi. org/10.4315/0362-028X.JFP-11-175

Hricova, D., Stephan, R. y Zweifel, C. (2008). Electrolyzed water and its application in the food industry. Journal of Food Protection, 71, pp. 1934-1947. https://doi. org/10.4315/0362-028X-71.9.1934

Kaufmann-Horlacher, I., Scherbaum, E., Stroher-Kolberg, D. y Wildgrube, C. (2014). Chlorate residues in plantbased food: Origin unknown. CVUA Sttutgart. [En línea]. Disponible en: http://www.cvuas.de/pub/beitrag. asp?subid=1\&ID=1854\&Pdf=No
Kitis, M. (2004). Disinfection of wastewater with peracetic acid: a review. Environment International, 30, pp. 47-55. https://doi.org/10.1016/S01604120(03)00147-8

López-Gálvez, F., Allende, A., Selma, M. V. y Gil, M. I. (2009). Prevention of Escherichia coli cross-contamination by different commercial sanitizers during washing of fresh-cut lettuce. International Journal of Food Microbiology, 133 (1-2), pp. 167-171. https://doi.org/10.1016/j. ijfoodmicro.2009.05.017

López-Gálvez, F., Allende, A., Truchado, P., Martínez-Sánchez, A., Tudela, J. A., Selma, M. V. y Gil, M. I. (2010). Suitability of aqueous chlorine dioxide versus sodium hypochlorite as an effective sanitizer for preserving quality of freshcut lettuce while avoiding by-product formation. Postharvest Biology and Technology, 55 (1), pp. 53-60. https://doi. org/10.1016/j.postharvbio.2009.08.001

López-Gálvez, F., Posada-Izquierdo, G. D., Selma, M. V., Pérez-Rodríguez, F., Gobet, J., Gil, M. I. y Allende, A. (2012). Electrochemical disinfection: An efficient treatment to inactivate Escherichia coli 0157:H7 in process wash water containing organic matter. Food Microbiology, 30 (1), pp. 146-156. https://doi. org/10.1016/j.fm.2011.09.010

López-Gálvez, F., Tudela, J. A., Allende, A. y Gil, M. I. (2019). Microbial and chemical characterization of commercial washing lines of fresh produce highlights the need for process water control. Innovative Food Science and Emerging Technologies. Innovative Food Science and Emerging Technologies, 51, pp. 211-219. https://doi.org/10.1016/j. ifset.2018.05.002

Luo, Y., Nou, X., Yang, Y., Alegre, I., Turner, E., Feng, H., Abadias, M. y Conway, W. (2011). Determination of free chlorine concentrations needed to prevent Escherichia coli 0157:H7 cross- contamination during fresh.cut 
produce wash. Journal of Food Protection, 74 (3), pp. 352-358. https://doi. org/10.4315/0362-028X.JFP-10-429

Manzocco, L., Ignat, A., Anese, M., Bot, F., Calligaris, S., Valoppi, F. y Nicoli, M. C. (2015). Efficient management of the water resource in the fresh-cut industry: Current status and perspectives. Trends in Food Science and Technology, 46, pp. 286-294. https://doi.org/10.1016/j. tifs.2015.09.003

Mena, K. D. (2006). Produce quality and foodborne disease: Assessing water's role. En: James, J. A. (ed.). Microbial hazard identification in fresh fruits and vegetables. Hoboken, NJ: Wiley, pp. 95-114. https://doi.org/10.1002/0470007761. ch4

Munther, D. y Wu, J. (2013). Enhanced surveillance on food-borne disease outbreaks: Dynamics of cross-contamination in biocidal wash procedure. Journal of Theoretical Biology, 321, pp. 28-35. https://doi.org/10.1016/j. jtbi.2012.12.024

Ölmez, H.y Kretzschmar, U. (2009). Potential alternative disinfection methods for organic fresh-cut industry for minimizing water consumption and environmental impact. LWT- Food Science and Technology, 42, pp. 686-693. https:// doi.org/10.1016/j.Iwt.2008.08.001

Selma, M. V., Allende, A., López-Gálvez, F., Conesa, M. A. y Gil, M. I. (2008a).
Heterogeneous photocatalytic disinfection of wash waters from the fresh-cut industry. Journal of Food Protection, 71, pp. 286-292. https://doi. org/10.4315/0362-028X-71.2.286

Selma, M. V., Allende, A., López-Gálvez, F., Conesa, M. A. y Gil, M. I. (2008b). Disinfection potential of ozone, ultraviolet- $\mathrm{C}$ and their combination in wash water for the fresh-cut vegetable industry. Food Microbiology, 25 (6), pp. 809-814. https://doi.org/10.1016/j. fm.2008.04.005

United States Food and Drug Administration (USFDA) (2001). Analysis and Evaluation of Preventive Control Measures for the Control and Reduction/Elimination of Microbial Hazards on Fresh and Fresh-cut Produce. Report of the Institute of Food Technologists. IFT/FDA Contract No. 3.

Van Haute, S., López-Gálvez, F., GómezLópez, V. M., Eriksson, M., Devlieghere, F., Allende, A. y Sampers, I. (2015). Methodology for modeling the disinfection efficiency of fresh-cut leafy vegetables wash water applied on peracetic acid combined with lactic acid. International Journal of Food Microbiology, 208, pp. 102-113. https://doi. org/10.1016/j.ijfoodmicro.2015.05.020

\section{Textos legales}

Directiva 98/83/CE del Consejo de 3 de noviembre de 1998 relativa a la calidad de las aguas destinadas al consumo humano. Diario Oficial de las Comunidades Europeas, 5 diciembre 1998. Disponible en https://eur-lex.europa.eu/legal-content/ES/ALL/?uri=celex\%3A31998L0083

Nota de la Comisión sobre la Guía para combatir los riesgos microbiológicos en frutas y hortalizas frescas en la producción primaria mediante una buena higiene. Diario Oficial de la Unión Europea, 23 mayo 2017. Disponible en https://eurlex.europa.eu/legal-content/ES/ALL/?u ri=CELEX\%3A52017XC0523\%2803\%29

Reglamento (CE) $n^{\circ}$ 852/2004 del Parlamento Europeo y del Consejo, de 29 de abril de 2004, relativo a la higiene de los productos alimenticios. Diario Oficial de la Unión Europea, 30 abril 2004. Disponible en https:// eur-lex.europa.eu/legal-content/ES/ ALL/?uri=CELEX\%3A32004R0852

\section{Recursos en línea}

Chlorine and its oxides: Chlorate and perchlorate review. AHDB. [En línea]. Disponible en: https://ahdb.org.uk/ cp-154a-chlorine-and-its-oxides-chlorate-perchlorate-review

Whitaker, B. Key Learnings from CPS Wash Water Symposium. [En línea]. Disponible en: https://www.centerforproducesafety.org/amass/documents/ document/118/Key\%20Learnings\%20 from $\% 20$ CPS $\% 20$ Wash $\% 20$ Water $\% 20$ Symposium\%20January\%202013.pdf 